\title{
PRELIMINARY RESULTS FROM PROTOTYPE NIOBIUM CAVITIES FOR THE JLAB AMPERE-CLASS FEL*
}

\author{
P. Kneisel", G. Ciovati, R. Bundy, B. Clemens, D. Forehand, B. Golden, S. Manning, B. Manus, F. \\ Marhauser, R. Overton, R.A.Rimmer, G. Slack,L. Turlington and H. Wang, Jefferson Lab, 12000 \\ Jefferson Ave, Newport News, VA 23606, USA
}

\begin{abstract}
In a previous paper the cavity [1] design for an Ampere-class cryomodule was introduced. We have since fabricated a $1500 \mathrm{MHz}$ version of a single cell cavity with waveguide couplers for HOM and fundamental power, attached to one end of the cavity, a 5-cell cavity made from large grain niobium without couplers and. a 750 $\mathrm{MHz}$ single cell cavity without endgroups to get some information about obtainable Q-values, gradients and multipacting behavior at lower frequency.

This contribution reports on the various tests of these cavities.
\end{abstract}

\section{INTRODUCTION}

For the next generation of compact high power FEL's a cryomodule is required that is capable of accelerating beam currents up to Ampere levels. Such a new module is under consideration at Jlab with emphasis on the development of schemes for strong HOM damping, absorption of high HOM power and feeding large amounts of fundamental mode power to the cavities. For compactness of the module a rather high real-estate gradient in the cavities is desirable. The concept of the module, based on waveguide coupling for input power and HOM power was introduced in Ref. [1]. Several single cell and one five cell cavity of the chosen shape have been fabricated and tested at JLab and this contribution reports about the experiences gained with these cavities.

\section{PROTOTYPE CAVITIES}

A picture of the prototype cavities is shown in Fig. 1. They are: a single cell cavity without end groups, a single cell cavity with a three waveguide endgroup for HOMdamping and input power, a 5-cell cavity without end groups made from large grain niobium - all these cavities are resonating at $1497 \mathrm{MHz}$ - and a scaled single cell cavity at $750 \mathrm{MHz}$. The rationale for fabricating and testing these different cavities will be discussed below. Table 1 summarizes the cavity parameters. The cavity performance goals for the compact cryo-module are an accelerating gradient of $\mathrm{E}_{\text {acc }}=16.7 \mathrm{MV} / \mathrm{m}$ at a Q-value of $\mathrm{Q}_{0}=8 \times 10^{9}$.

* This manuscript has been authored by Jefferson Science Associates, LLC under U.S. DOE Contract No. DE-AC05-06OR23177. The U.S. Government retains a non-exclusive, paid-up, irrevocable, world-wide license to publish or reproduce this manuscript for U.S. Government purposes.

"kneisel@jlab.org

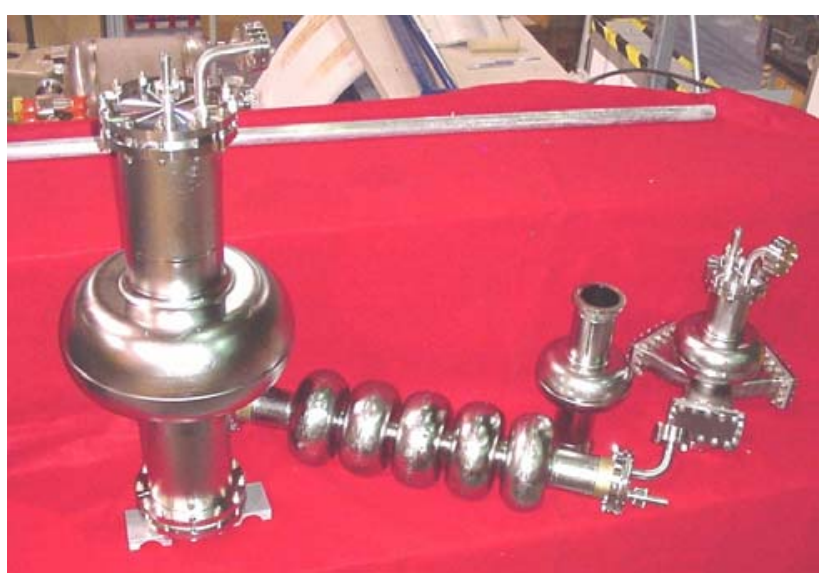

Figure 1: Collection of Prototype cavities.

Table 1: Prototype cavities parameters.

\begin{tabular}{|l|l|l|l|}
\hline $\begin{array}{l}\text { Cavity/ } \\
\text { Frequency } \\
\text { (MHz) }\end{array}$ & $\begin{array}{l}\mathbf{1 / L} \sqrt{ } \mathbf{R} / \mathbf{Q} \\
(\boldsymbol{\Omega})\end{array}$ & $\mathbf{E}_{\mathbf{p}} / \mathbf{E}_{\text {acc }}$ & $\begin{array}{l}\mathbf{B}_{\mathbf{p}} / \mathbf{E}_{\text {acc }} \\
{[\mathbf{m T} /(\mathbf{M V} / \mathbf{m})]}\end{array}$ \\
\hline 1-cell/1497 & 104.1 & 2.19 & 4.09 \\
\hline $\begin{array}{l}\text { 1-cell with } \\
\text { WG/1497 }\end{array}$ & 101.4 & 2.63 & 4.30 \\
\hline 5-cell/1497 & 45.8 & 2.5 & 4.27 \\
\hline 1-cell/750 & 50.9 & 2.24 & 4.18 \\
\hline
\end{tabular}

All cavities have been fabricated from high thermal conductivity niobium with RRR-values $\geq 250$; the single cell with waveguides and the 5-cell cavity were made from large grain niobium, for the other prototypes fine grain niobium was used. Fabrication utilized standard procedures: deep drawing of half cells, trimming for electron beam welding, EBW with full penetration welds at the equators and inside-outside welds at the irises (either dumbbells or beam pipe welds), app. 100 micron of material removal by BCP (1:1:1), hydrogen degassing at $600{ }^{\circ} \mathrm{C}$ for $10 \mathrm{hrs}$ in ultrahigh vacuum and final surface treatment again by BCP, where app. 60 micron of additional material was removed. High pressure ultrapure water rinsing for one to several hours was carried out prior to class 10 clean room assembly after drying of the cavities for 12 hours in the class 10 clean room. Usually the cavity vacuum reached the low $10^{-8} \mathrm{mbar}$ range after evacuation on the cryogenic test stand for 12 hrs. 


\section{Single Cell Cavity-1497 MHz}

This cavity was fabricated and tested to evaluate the performance of this cavity shape: because of the flat side walls of the cavity there were some concerns about being able to chemically polish and high pressure rinse contamination-free the cavity and whether a predicted multipacting barrier, even though rather soft, would be a problem. The cavity performed quite well as shown in Fig. 2 after some initial difficulties with field emission in the first tests. After post-purification with $\mathrm{Ti}$ at $1250{ }^{\circ} \mathrm{C}$ for 3 hrs , additional removal of app. $30 \mu \mathrm{m}$ by BCP and “in-situ” baking at $120^{\circ} \mathrm{C}$ for 12 hrs the performance as shown in Fig. 2 was obtained. The quench limit was not reached because of rf power limitations due to the modest Q-drop, which was not totally eliminated after the "insitu" baking, which is common for fine grain niobium after BCP treatment.

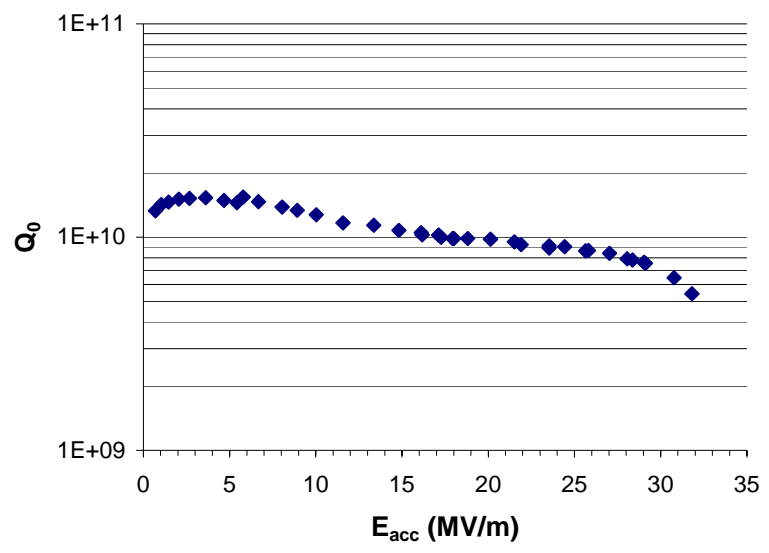

Figure 2: Performance of single cell prototype cavity measured at $2 \mathrm{~K}$.

\section{Single Cell cavity with End Group[Figure3]}

The objectives for fabricating and testing this cavity were similar to the ones for the single cell cavity, except for the more complex shape. Exploring the capability to clean and to achieve high Q-values and gradients were the major goals. There was/is some concern about the rather large amount of fundamental power propagating out of the waveguides. This may require much longer niobium waveguide sections as presently planned for in the cryomodule. In addition the manufacturing of the waveguide assembly was another major milestone, which had to be demonstrated.

In the first test after standard treatment as discussed above the cavity showed a strong degradation of the Qvalue with increasing field as shown in Fig. 4. This behavior, which is an indication of heating ( the additional resistance is proportional to $\mathrm{H}^{2}$ ) did not change after "insitu” baking or additional material removal carried out in test \#2 and test \#3, respectively. After post-purification with $\mathrm{Ti}$ at $1250{ }^{\circ} \mathrm{C}$ for $3 \mathrm{hrs}$ and additional BCP, the result indicated in Fig. 3 was obtained. The Q-degradation, even though its onset has shifted to higher gradients, is

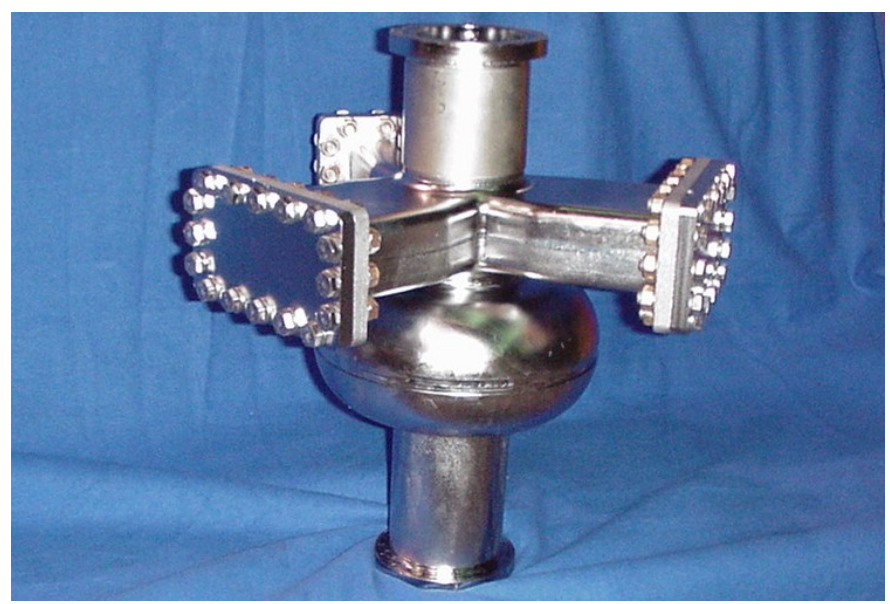

Figure 3: Single Cell Cavity with End Group

still present and might be an indication of losses in the shortened waveguide sections.

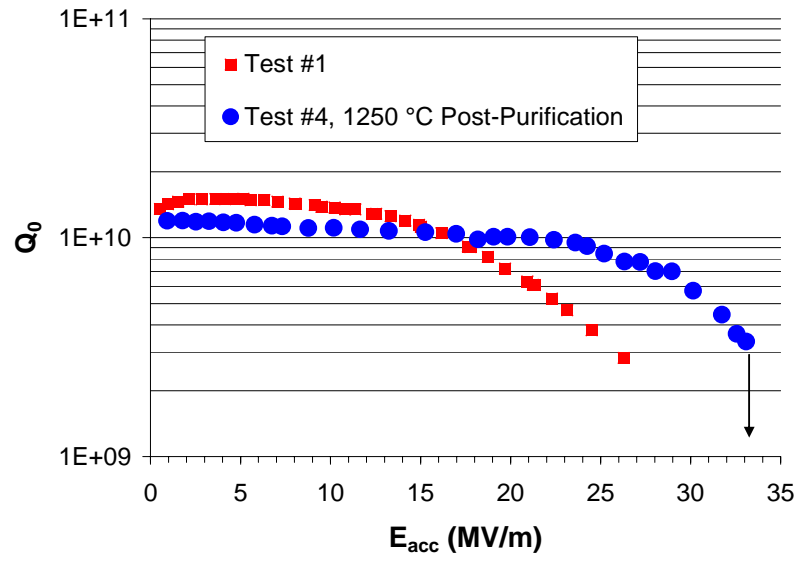

Figure 4: Performance of single cell 1-Amp cavity with waveguide assembly, measured at $2 \mathrm{~K}$.

\section{Five Cell Prototype Cavity - $1497 \mathrm{MHz}$}

This cavity was built from large grain niobium supplied by CBMM. The objective was to verify or exceed the design goals for the $1 \mathrm{Amp}$ project and to make sure that multipacting was not present in a multi-cell structure. The results from all tests are summarized in Fig. 5.

Obviously it was more difficult to obtain/exceed the design goals for this cavity. Initially insufficient amounts of material were removed from the surface, the additional BCP in test \#2 (50 micron) improved the performance; however only after a post-purification heat treatment the design goals were exceeded. 


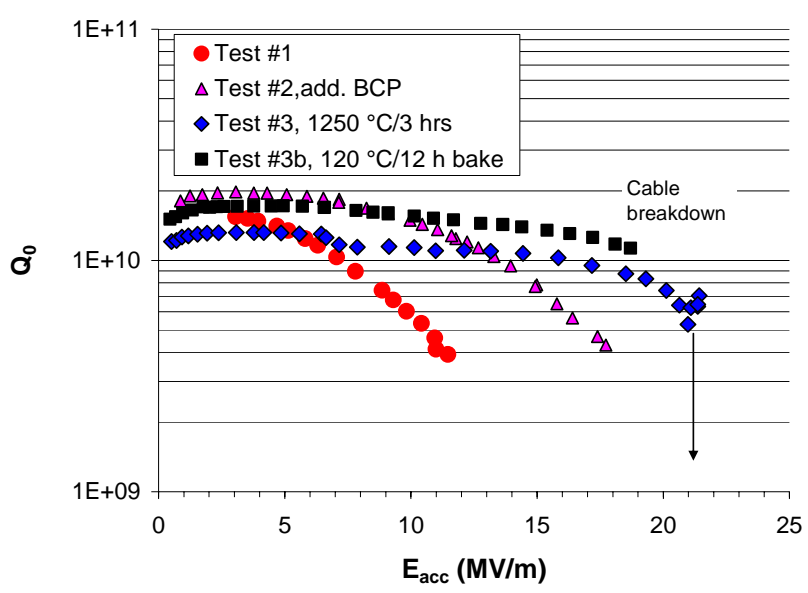

Figure 5: Summary of tests carried out with the 5-cell cavity at $2 \mathrm{~K}$.

\section{Single Cell Cavity- $750 \mathrm{MHz}$}

This cavity is a scaled version of the $1497 \mathrm{MHz}$ shape. The objective here was to explore, whether this lower frequency version would show different behavior in performance than the $1497 \mathrm{MHz}$ model. This exploration has some significance since the eventual high current cryo-module will be built at this lower frequency.

The result of the first and only test carried out with this cavity is shown in Fig. 6: no multipacting was encountered in this test and in addition, the highest obtained gradient of $\mathrm{E}_{\mathrm{acc}} \sim 27 \mathrm{MV} / \mathrm{m}$ was not limited by a quench, but rather by the onset of the Q-drop.

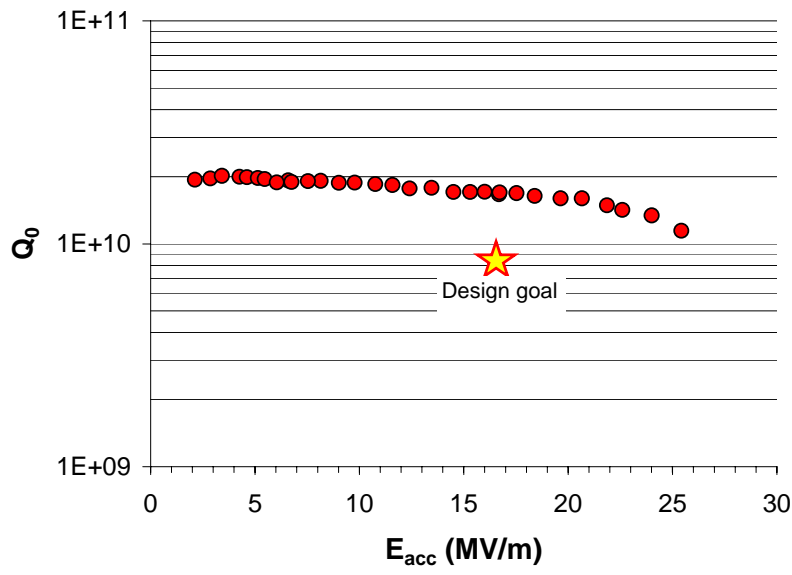

Figure 6: $\mathrm{Q}_{0}$ vs $\mathrm{E}_{\text {acc }}$ for $750 \mathrm{MHz}$ single cell cavity measured at $2 \mathrm{~K}$.

\section{SUMMARY}

All four prototype cavities performed well and exceeded the design goals. With these test results the stage is set for fabricating and testing fully equipped 5cell cavities - initially at $1497 \mathrm{MHz}$. One possible difficulty will be losses in the waveguides for HOM - and fundamental power as indicated above. This will require an extension of niobium waveguide pieces outside the He- vessel, cooled and kept superconducting by conduction or an additional helium circuit. It will be learnt in the near future whether this would be necessary, when the 5-cell cavities, presently being fabricated, will be available for testing.

\section{ACKNOWLEDGEMENTS}

We would like to thank all our colleagues, who supported this work.

\section{REFERENCES}

[1] R. A. Rimmer et al., "The JLab Ampere-Class Cryomodule Conceptual Design” EPAC’06, Edinburgh, June 2006, p. 490. 